\title{
Quantitative Assessment of Right Ventricular Size and Function with Multiple Parameters from Artificial Intelligence-Based Three-Dimensional Echocardiography: A Comparative Study with Cardiac Magnetic Resonance
}

Ying Zhu ${ }^{1}$, Yuwei bao ${ }^{1}$, Kangchao Zheng ${ }^{1}$, Wei Zhou ${ }^{1}$, Zhang Jun ${ }^{1}$, Ruiying Sun ${ }^{1}$, You-Bin Deng ${ }^{2}$, Liming Xia ${ }^{1}$, and Yani Liu ${ }^{3}$

${ }^{1}$ Affiliation not available

${ }^{2}$ Huazhong Univ Sci

${ }^{3}$ Tongji Hospital of Tongji Medical College of Huazhong University of Science and Technology

August 19, 2021

\begin{abstract}
Abstract Aims: This study aimed to explore the validation and the diagnostic value of multiple right ventricle (RV) volumes and functional parameters parameters derived from a novel artificial intelligence (AI)-based three-dimensional echocardiography (3DE) algorithm compared to cardiac magnetic resonance (CMR). Methods and Results: 51 patients with a broad spectrum of clinical diagnoses were finally included in this study. AI-based RV 3DE was performed in a single-beat HeartModel mode within 24 hours after CMR. Whether in the entire population or the patients with moderate and poor image quality, RV volumes and right ventricular ejection fraction (RVEF) measured by AI-based 3DE showed a statistically significant correlation with the corresponding CMR analysis $(\mathrm{P}<0.05$ for all). The Bland-Altman plots indicated that these parameters were slightly underestimated by AI-based 3DE. Based on CMR derived RVEF $<45 \%$ as RV dysfunction, end-systolic volume (ESV), end-systolic volume index (EDVi), stroke volume (SV), and RVEF showed great diagnostic performance in identifying RV dysfunction, as well as some non-volumetric parameters, including tricuspid annular systolic excursion (TAPSE), fractional area change (FAC), RV septum and free-wall longitudinal strains (LS) ( $\mathrm{P}<0.05$ for all). The cutoff value was $43 \%$ for RVEF with a sensitivity of $94 \%$ and specificity of $67 \%$. Conclusion: AI-based 3DE provide rapid and accurate quantitation of the RV volumes and function with multiple parameters. Both volumetric and non-volumetric measurements derived from AI-based 3DE contributed to the identification of the RV dysfunction, even in the patients without excellent image quality of RV 3DE. Keywords: artificial intelligence, three-dimensional echocardiography, right ventricle, multiple parameters
\end{abstract}

Quantitative Assessment of Right Ventricular Size and Function withMultiple Parameters from Artificial Intelligence-Based Three-Dimensional Echocardiography: A Comparative Study with Cardiac Magnetic Resonance

Ying Zhu ${ }^{1}$, MD, PhD, Yuwei Bao ${ }^{1}$, MD, Kangchao Zheng ${ }^{1}$, MD,Wei Zhou ${ }^{1}$, MD, PhD, Zhang Jun ${ }^{1}$, MD, $\mathrm{PhD}$, Ruiying Sun ${ }^{1}, \mathrm{MD}$, Youbin Deng ${ }^{1}, \mathrm{MD}, \mathrm{PhD}$, Liming Xia ${ }^{2}, \mathrm{MD}, \mathrm{PhD}$, Yani $\mathrm{Liu}^{1}, \mathrm{MD}, \mathrm{PhD}$

${ }^{1}$ Department of Medical Ultrasound, Tongji Hospital, Tongji Medical College, Huazhong University of Science and Technology, 1095 Jiefang Road, Wuhan 430030, China

${ }^{2}$ Department of Radiology, Tongji Hospital, Tongji Medical College, Huazhong University of Science and Technology, 1095 Jiefang Road, Wuhan 430030, China 
Corresponding Author: Yani Liu, From Dept. of Medical Ultrasound, Tongji Hospital, Tongji Medical College, Huazhong University of Science and Technology, 1095 Jiefang Road, Wuhan, 430030, PR China. Phone: +8613886062141

E-mail:yani.liu@tjh.tjmu.edu.cn; yani.liu@163.com

There was no conflicts of interest to declare.

Type of manuscript: Original contribution

\section{Abstract}

Aims: This study aimed to explore the validation and the diagnostic value of multiple right ventricle (RV) volumes and functional parameters parameters derived from a novel artificial intelligence (AI)-based three-dimensional echocardiography (3DE) algorithm compared to cardiac magnetic resonance (CMR).

Methods and Results: 51 patients with a broad spectrum of clinical diagnoses were finally included in this study. AI-based RV 3DE was performed in a single-beat HeartModel mode within 24 hours after CMR. Whether in the entire population or the patients with moderate and poor image quality, RV volumes and right ventricular ejection fraction (RVEF) measured by AI-based 3DE showed a statistically significant correlation with the corresponding CMR analysis $(\mathrm{P}<0.05$ for all). The Bland-Altman plots indicated that these parameters were slightly underestimated by AI-based 3DE. Based on CMR derived RVEF $<45 \%$ as RV dysfunction, end-systolic volume (ESV), end-systolic volume index (EDVi), stroke volume (SV), and RVEF showed great diagnostic performance in identifying RV dysfunction, as well as some non-volumetric parameters, including tricuspid annular systolic excursion (TAPSE), fractional area change (FAC), RV septum and free-wall longitudinal strains (LS) $(\mathrm{P}<0.05$ for all). The cutoff value was $43 \%$ for RVEF with a sensitivity of $94 \%$ and specificity of $67 \%$.

Conclusion: AI-based 3DE provide rapid and accurate quantitation of the RV volumes and function with multiple parameters. Both volumetric and non-volumetric measurements derived from AI-based 3DE contributed to the identification of the RV dysfunction, even in the patients without excellent image quality of RV 3DE.

Keywords: artificial intelligence, three-dimensional echocardiography, right ventricle, multiple parameters

\section{Introduction}

Rapid and accurate assessment of the right ventricle (RV) is a great challenge because of its complex anatomy and mechanics [1-2]. Although the role of the RV was often underestimated, many studies demonstrated the RV dysfunction could independently predict the worse outcomes in various cardiac conditions, such as pulmonary arterial hypertension (PAH), heart failure, and congenital heart disease [2-4]. There is also growing evidence that RV size and function are critical determinants in the treatment decision-making and perioperative complications for patients undergoing cardiac or noncardiac surgeries [4-6].

Currently, cardiac magnetic resonance (CMR) is regarded as the gold standard for quantifying RV volume and function because of its excellent spatial resolution and three-dimensional volume rendering [2,7]. However, limited availability, high cost, and long image acquisition time hampered its wide use in different clinical settings. Besides, its performance would be reduced seriously in patients with arrhythmia or critically ill patients who cannot hold breath during image acquisition[2,7]. Echocardiography is the most commonly used imaging technique to assess cardiac function. However, the conventional linear and area measurements by two-dimensional echocardiography (2DE) often failed to give accurate estimates of RV volume [2,8-9]. It is not recommended to calculate the right ventricular ejection fraction (RVEF) derived from RV volumes on $2 \mathrm{DE}[9]$.

Previous studies have confirmed that RV volumes and RVEF from three-dimensional echocardiography (3DE) were accurate and reproducible compared to CMR [10-12]. However, conventional 3DE software still requires manual analysis and is time-consuming and skill demanding, and therefore, it is unacceptable in a busy 
echocardiographic laboratory [10-12]. A novel artificial intelligence (AI)-based 3DE algorithm, named 3D Auto RV, holds the promise to provide an ideal solution for fast and convenient quantification of RV volume and function [13]. A recent study verified this algorithm with CMR in RV volumes and EF measurements [13]. However, the diagnostic ability of AI-based 3DE in the identification of RV systolic dysfunction was not elucidated. This study performed a comprehensive assessment of RV size and function with multiple volumetric and non-volumetric parameters using the new AI-based 3DE algorithm. We also analyzed the diagnostic value of these measurements regarding their sensitivity and specificity in detecting patients with impaired RV function (CMR-derived $\mathrm{EF}<45 \%$ ).

\section{Materials and Methods}

\section{Study Population}

From January 2021 to February 2021, 54 consecutive patients (31 men and 23 women, median age [range]: $52[34-59]$ y) referred for a clinically indicated CMR were recruited in this study. RV volumes and function were measured using the AI-based 3DE software within 24 hours after CMR. Reliable CMR analysis was not completed in 3 critically ill patients due to motion artifact. A total of 51 patients were finally included in this study. The Ethics Committee approved this study, and informed consent was obtained from all patients before the study.

\section{RV 3DE Image Acquisition}

Transthoracic echocardiography was performed by two experienced doctors using an EPIQ CVx system (Philips Healthcare, Andover, MA), equipped with an X5-1 transducer. RV 2DE was imaged on apical RV focused four-chamber view according to the guidelines from the American Society of Echocardiography (ASE) [9]. Then, RV 3DE were acquired in a single-beat HeartModel mode. Imaging depth and sector width was controlled to obtain the optimized $3 \mathrm{DE}$ frame rate. The entire RV and left ventricle (LV) chambers should be encompassed during the imaging acquisition. All three-dimensional (3D) data was stored digitally and analyzed with the machine built-in software.

\section{AI-based RV 3DE Analysis}

Before the image analysis, image quality was evaluated on two-dimensional (2D) apical four-chamber, inflowoutflow, and short-axis views extracted from RV 3DE data. The right ventricular wall was divided into 17 segments, five on the short axis view, six on the four-chamber view, and six on the inflow-outflow tract view [13-14]. Image quality was graded as poor (defined as RV endocardial border were invisible in $>2$ continuous segments on at least one view), moderate (between the poor and the good images), or good (full RV endocardial visualization on three views) in each patient.

All measurements were automatically obtained using the machine built-in software (3D Auto RV, QLAB, version 13, Philips Healthcare). After selecting RV 3DE data in HeartModel mode, it continuously identifies and traces the RV endocardium throughout the cardiac cycle in multiple tomographic views. Then 3D RV end-diastolic volume (EDV), end-diastolic volume index (EDVi), end-systolic volume (ESV), and endsystolic volume index (ESVi), stroke volume (SV) and RVEF were automatically calculated (Fig. 1a). The conventional 2D RV non-volumetric parameters, including tricuspid annular plane systolic excursion (TAPSE), fraction area change (FAC), and RV longitudinal strain (LS), were also presented side by side (Fig. 1b). This software permits manual editing of the RV endocardial border when some parts of the RV wall did not track adequately. All the RV volumes and functional measurements were recorded for each patient.

\section{CMR Imaging and RV Quantification Analysis}

All subjects underwent CMR examination on a 3.0T scanner (Skyra, Siemens, Healthineers, Erlangen, Germany) using an 18-channel cardiac coil, head-first in a supine position. The dynamic imaging protocol included the RV short-axis cine (from apex to tricuspid annulus) and four-chamber cine with electrocardiograph gating during breath-hold. 
CMR images were analyzed offline using commercial software CVI42 (Circle Cardiovascular Imaging, Inc.), performed by an experienced radiologist blinded to all 3DE information. During the analysis process, 6 to 12 short-axis slices on steady-state free precession (SSFP) images from the tricuspid valve insertion point to the apex to encompass the entire RV were obtained. In every slice, the endocardial contours were manually traced at end-diastole (the image phase with the largest cavity area) and end-systole (the image phase with the smallest cavity area). The disk summation was used to calculate RV volume indices, including EDV, EDVi, ESV, and ESVi. RVEF was calculated using the standard formula: $\mathrm{EF}=(\mathrm{EDV}-\mathrm{ESV}) / \mathrm{EDV}$. The measurements were repeated three times for each patient, and the final measurements were defined as the average value of three repeated measurements.

\section{Reproducibility}

To assess the reproducibility and accuracy of the research results, we randomly selected 20 patients' 3DE images. The images were analyzed by two experienced independent researchers who were blinded to the patients' information and CMR results. The inter-observer and intra-observer variabilities for all the RV measurements were analyzed using the intra-class correlation coefficient (ICC).

\section{Statistical analysis}

Statistical analysis was accomplished using the SPSS software package (version 22.0; SPSS, Chicago, III). Categorical variables were expressed as percentages and were statistically analyzed by the chi-square test or Fisher's exact test. The Shapiro-Wilk test was used to verify whether continuous variables met the normal distribution. If continuous variables obeyed a normal distribution, the paired $t$-test was used to compare the two groups' parameters. Continuous values that did not obey the normal distribution were analyzed using the Mann-Whitney U test and are presented as median (50th) values and interquartile ranges (25th and 75th). Spearman linear correlation and Bland-Altman analysis were performed to compare the RV measurements derived from AI-based 3DE with CMR. Receiver operating characteristic (ROC) curve analysis was performed to investigate the diagnostic value of RV parameters from 3DE in identifying RV dysfunction. Comparison of areas under the curve (AUC) was performed using the method of DeLong in MedCalc. All results were considered statistically significantly different at $\mathrm{P}<0.05$.

\section{Results}

\section{Clinical Characteristics of Study Population}

A total of 51 patients with sinus rhythm were finally included in this study. Table 1 showed the clinical characteristics of the study population. Since the unselected patients referred for CMR were enrolled, this study encompassed a broad spectrum of cardiac diagnoses.

\section{Image Quality Assessment of RV 3DE}

Of the 18 patients with good image quality, the fully automated analysis was completed in $12(66.7 \%)$ patients. For 27 patients with moderate image quality, the fully automated analysis was completed in 7 (25.9\%) patients, and manual editing was needed in 20 patients. The fully automated analysis failed in the 6 patients with poor images, and a more manual correction was required during the analysis procession.

The analysis time for AI-based 3DE RV quantification was significantly dependent on image quality. A fully automated analysis was finished in 19 patients in $15 \pm 1$ seconds. With manual editing performed when needed, AI-based 3DE RV analysis was completed in $100 \pm 12$ seconds in patients with good quality images, $105 \pm 6$ seconds with moderate, and $162 \pm 3$ seconds with poor quality images $(\mathrm{P}<0.05$ for any two groups). The comprehensive RV volumes and RVEF analysis for CMR imaging were generally completed in $448 \pm 25$ seconds.

Table 2 and Fig. 2 showed the comparisons of RV Volumes and RVEF between AI-based 3DE and CMR. All the measurements from both modalities showed statistically significant correlations in the whole study population $(\mathrm{P}<0.05$ for all). Although the AI-based 3DE algorithm slightly underestimated RV volumes and RVEF compared with CMR, the Bland-Altman plots indicated that the differences obtained by the two 
modalities distributed around the mean of the difference (Fig. 2). It's worth noting statistically significant correlations between the two modalities were also found for all the RV measurements in the moderate and poor image quality group $(\mathrm{P}<0.05$ for all). However, compared with the good image group, a lower correlation value and greater limits of agreement (LOA) for the measurements except EDVi were found in the moderate and poor image quality group (Table 2).

\section{Identifying RV Dysfunction Using AI-based 3DE Quantitative Parameters in comparison with CMR}

Based on CMR as the gold standard, RV dysfunction was defined as CMR-derived RVEF $<45 \%$ [10]. Table 3 summarized the clinical characteristics and the quantitative parameters derived from AI-based 3DE in patients with CMR-derived RVEF [?] $45 \%$ and;45\%.

In the entire study population, significant differences were found in ESV, ESVi, SV, EF, TAPSE, FAC, RV septum LS, and RV free-wall LS between the normal and the reduced RV function groups $(\mathrm{P}<0.05$ for all) (Table 3). As shown in Figure 3 and Table 4, most of the volumetric and non-volumetric parameters from AI-based 3DE showed good diagnostic performance in identifying RV dysfunction with CMR-derived RVEF $; 45 \%$ ( $\mathrm{P}<0.05$ for all). Of these multiple quantitative indices, RVEF from AI-based 3DE was superior to the others at a value of $43 \%$ diagnosed RV dysfunction with $94 \%$ sensitivity and $67 \%$ specificity whereas the there was no statistically significance $(\mathrm{P}>0.05)$. Even in patients with moderate and poor RV 3DE images, ESVi, RVEF, FAC, and RV septum LS can be used to distinguish normal from abnormal RV function with acceptable sensitivity and specificity $(\mathrm{P}<0.05$ for all) (Table 4$)$.

\section{Reproducibility}

In a random sample of 20 people, all RV volumes and functional parameters exhibited excellent intraand interobserver reproducibility. The detailed ICCs for interobserver and intraobserver variability were presented in Table 5.

\section{Discussion}

This study performed an automatic assessment of RV size and function with multiple quantitative parameters using AI-based 3DE. Our findings indicated that the analysis process for AI-based 3DE was significantly dependent on image quality. On the whole, RV volumes and RVEF measured by AI-based 3DE showed a good correlation with CMR, with a slight underestimation. Based on CMR-derived RVEF $<45 \%$ as RV dysfunction, RVEF showed an excellent performance in identifying the reduced RV function at a cut value of $43 \%$ with $94 \%$ sensitivity and $67 \%$ specificity $(\mathrm{AUC}=0.854, \mathrm{p}<0.001)$. Some non-volumetric parameters, such as FAC and RVLS, can also be used to distinguish normal from abnormal RV function, even in patients without excellent RV 3DE images.

For a long time, the importance of RV in cardiac performance was often neglected in comparison to the left ventricle [1]. With the development of non-invasive imaging modalities, accumulating evidence indicated the crucial role of RV in determining functional status and prognosis in universal clinical conditions [3-7, 15-16]. In patients with pulmonary embolism, PAH, and arrhythmogenic RV dysplasia, the dilated RV was a strong predictor of morbidity and mortality [3,15-16]. A single-center study by Chou et al. pointed out RV dysfunction was associated independently with a higher incidence of postoperative major cardiovascular events and more extended hospital stays [6]. Therefore, the precise and reliable quantitative assessment of RV size and function is widely required to diagnose and manage cardiovascular disease in different clinical settings, such as emergency room, intraoperative and perioperative care.

CMR is currently regarded as the gold standard for accurate assessment of RV volumes and function [2]. Still, its high cost, time-consuming and limited availability preclude its wide application as a routine method of choice. Echocardiography is the most commonly used cardiac imaging modalities in clinical practice. With the improvement of 3DE, a large of previous studies confirmed that 3DE could overcome the challenges related to the complex RV geometry and was superior to $2 \mathrm{DE}$ in RV analysis [12, 17-20]. Although 3DE was recommended by ASE guidelines as the primary method for quantifying RV volumes and EF [9], this 
effective technique has not been widely used in clinical practice until now. The main limiting factor is the cumbersome manual tracing of the RV endocardial, which is time-consuming, skill demanding, and relatively poor reproducibility [13].

Artificial intelligence (AI) has the potential to improve the analysis and interpretation of medical images to a new stage compared with previous algorithms [21]. A novel AI-based 3DE algorithm has achieved significant success in fast measuring the dynamic left ventricle and atrium volumes and accurately analyzing ejection/filling parameters [22]. Recently, the machine-learning algorithm was also developed for automated RV quantitative analysis on 3DE [13, 23]. Consistent with Genovese et al.'s results[13], the present study confirmed that fully automatic RV analysis could be accurately realized in $37 \%$ of unselected patients in nearly 15 seconds. After manual correction for unsatisfying RV endocardial tracing, all the volumetric parameters and EF derived from AI-based 3DE showed an excellent correlation with CMR reference values. It's worth noting that the accuracy of AI-based 3DE algorithms depended on the 3DE image quality to some extent, and decreased image quality is often associated with a lower correlation and greater LOA for the measurements.

Compared with previous 3DE studies, a significant advantage of AI-based 3DE is the improved reproducibility of RV measurements [13,23]. In theory, a fully automated analysis was $100 \%$ reproducible. Even with manual correction in AI-based 3DE, excellent reproducibility was also found, as reflected by the coefficient of Variation $(\mathrm{CoV})<7 \%$ and ICC $>0.95$ for all the measurements in Genovese et al.'s study [13]. As for AI-based 3DE, image data was acquired in single-beat and high frame rate HeartModel mode. It is different from the full-volume image acquisition with a multiple-beat approach for conventional 3DE, which requires regular heart rhythm, stable probe position, and good breath-holding capability to avoid stitching artifacts [10-11]. In a study by Park et al., single-beat 3DE was confirmed to determine RV volume and systolic function with good accuracy and reproducibility, regardless of the rhythm status [10].

Along with previous RV volume studies on 3DE, the AI-based 3DE also inevitably underestimated the RV volumes, even in the patients with satisfying image quality $[10-11,13]$. We believed that the lower spatial resolution of $3 \mathrm{DE}$ could be the main reason for the unclear identification of the endocardial border, resulting in the smaller cavity contouring. With contrast-enhanced echocardiography, more accurate and reproducible 3D echocardiographic measurements of RV volumes can be obtained through improving the visualization of RV endocardial borders [24-25]. This approach may be beneficial in patients with suboptimal image quality.

In a broad spectrum of cardiovascular diseases, RVEF is related to future cardiovascular events and or all causes of death $[6,7,20,23]$. In our study, RVEF obtained from AI-based showed an excellent performance in identifying the reduced RV function at a cut value of $43 \%$ with $94 \%$ sensitivity and $67 \%$ specificity (AUC $=0.854, \mathrm{p}<0.001)$. Another study found that RVEF from AI-based 3DE was significantly associated with outcomes, providing accurate information regarding RV function and risk for adverse outcomes [23]. In addition to RVEF, some non-volumetric parameters, including RV FAC, TAPSE, and RV longitudinal strain, have also been proposed for RV functional assessment in recent years [7, 26]. A recent study developed a machine learning-based algorithm that could detect low RV function based on clinical and 2D echocardiographic parameters, including RVEF, TAPSE, S', and FAC [27]. With an AI-based algorithm, Liu et al. confirmed the feasibility of RV global longitudinal strain (GLS) on 2DE to assess intraoperative RV function [28]. In addition to the 3D volumetric parameters, AI-based 3DE could quickly provide the non-volumetric parameters of RV based on 2D four-chamber view automatically extracted from 3D data. As far as we know, our study firstly explored the diagnostic value of non-volumetric parameters from AI-based 3DE in determining RV dysfunction with a good sensitivity and specificity in comparison with CMR. Even in the patients with moderate and poor image quality, in whom RV free wall was often missing due to the location behind the thoracic cage and the shielding of pulmonary air, FAC and RV septum LS could identify RV dysfunction, but not the TAPSE and RV free-wall LS. Limitations

Several limitations in this study should be considered. First, the number of subjects in this study is still small. As for the unscreened patients referred for CMR, a wide range of RV volumes and RVEF were presented. Therefore, all the measurements obtained from AI-based 3DE and CMR did not obey the normal 
distribution. That might be why the weak correlation value of RVEF between AI-based 3DE and CMR, in which spearman linear correlation analysis was performed. Second, we did not compare the measurement differences among various experienced doctors based on AI-based 3DE algorithm. Third, the RV septum and free-wall LS, but not global RVLS was provided by the AI-based 3DE. Some studies indicated that the global RVLS could be more clinically relevant. Also, although contrast enhancement echocardiography contributes to improve the visualization of RV endocardial borders, especially in patients with suboptimal image quality, it was unavailable in the current AI-based 3DE algorithm.

\section{Conclusion}

This novel AI-based algorithm holds the promise to provide an ideal solution for fast and reliable quantification of the RV size and function with multiple RV volumetric and non-volumetric indices.

\section{Supplementary Data}

Table 1 showed the clinical characteristics of the study population. The detailed ICCs for interobserver and intraobserver variability in a random sample of 20 people, were presented in Table 5 .

\section{Acknowledgments}

This work was supported by Natural Science Foundation of Hubei Province in China (grant number 2019CFB691, 2019).

\section{References}

1. Sanz J, Sanchez-Quintana D, Bossone E, et al Anatomy, Function, and Dysfunction of the Right Ventricle: JACC State-of-the-Art Review. J AM COLL CARDIOL 2019, 73: 1463-1482.

2. Badano LP, Addetia K, Pontone G, et al. Advanced imaging of right ventricular anatomy and function. HEART 2020, 106: 1469-1476.

3. Vonk NA, Westerhof BE, Westerhof N. The Relationship Between the Right Ventricle and its Load in Pulmonary Hypertension. J AM COLL CARDIOL 2017, 69: 236-243.

4. Mrad AK, Burri M, Cleuziou J, et al. Preoperative predictability of right ventricular failure following surgery for Ebstein's anomaly. Eur J Cardiothorac Surg 2019,55: 1187-1193.

5. Bolat I. Preoperative Right Ventricular Echocardiographic Parameters Predict Perioperative Cardiovascular Complications in Patients Undergoing Non-Cardiac Surgery. HEART LUNG CIRC 2020, 29:1146-1151.

6. Chou J, Ma M, Gylys M, et al. Preexisting Right Ventricular Dysfunction Is Associated With Higher Postoperative Cardiac Complications and Longer Hospital Stay in High-Risk Patients Undergoing Nonemergent Major Vascular Surgery. J Cardiothorac Vasc Anesth 2019, 33: 1279-1286.

7. Smolarek D, Gruchala M, Sobiczewski W. Echocardiographic evaluation of right ventricular systolic function: The traditional and innovative approach. CARDIOL J 2017, 24: 563-572.

8. Addetia K, Maffessanti F, Muraru D, et al. Morphologic Analysis of the Normal Right Ventricle Using Three-Dimensional Echocardiography-Derived Curvature Indices. J Am Soc Echocardiogr 2018, 31: 614-623.

9. Lang RM, Badano LP, Mor-Avi V, et al. Recommendations for cardiac chamber quantification by echocardiography in adults: an update from the American Society of Echocardiography and the European Association of Cardiovascular Imaging. Eur Heart J Cardiovasc Imaging 2015,16: 233-270.

10. Park JB, Lee SP, Lee JH, et al. Quantification of Right Ventricular Volume and Function Using SingleBeat Three-Dimensional Echocardiography: A Validation Study with Cardiac Magnetic Resonance. J Am Soc Echocardiogr 2016, 29: 392-401.

11. Zhang QB, Sun JP, Gao RF, et al. Feasibility of single-beat full-volume capture real-time threedimensional echocardiography for quantification of right ventricular volume: validation by cardiac magnetic resonance imaging. INT J CARDIOL 2013, 168:3991-3995.

12. Nesser HJ, Tkalec W, Patel AR, et al. Quantitation of right ventricular volumes and ejection fraction by three-dimensional echocardiography in patients: comparison with magnetic resonance imaging and 
radionuclide ventriculography. Echocardiography. 2006,23:666-680.

13. Genovese D, Rashedi N, Weinert L, et al. Machine Learning-Based Three-Dimensional Echocardiographic Quantification of Right Ventricular Size and Function: Validation Against Cardiac Magnetic Resonance. J Am Soc Echocardiogr 2019, 32: 969-977.

14. Nowak-Machen M, Lang T, Schilling A, et al. Regional Right Ventricular Volume and Function Analysis Using Intraoperative 3-Dimensional Echocardiography-Derived Mesh Models. J Cardiothorac Vasc Anesth 2019,33: 1527-1532.

15. Park JS, Ahn J, Choi JH, et al. The predictive value of echocardiography for chronic thromboembolic pulmonary hypertension after acute pulmonary embolism in Korea. KOREAN J INTERN MED 2017,32: 85-94.

16. Mast TP, James CA, Calkins H, et al. Evaluation of Structural Progression in Arrhythmogenic Right Ventricular Dysplasia/Cardiomyopathy. JAMA CARDIOL 2017, 2: 293-302.

17. Liu YN, Deng YB, Liu BB, et al. Rapid and accurate quantification of right ventricular volume and stroke volume by real-time 3-dimensional triplane echocardiography. CLIN CARDIOL 2008, 31: 378-382.

18. Liu BY, Wu WC, Zeng QX, et al. The value of three-dimensional echocardiography in risk stratification in pulmonary arterial hypertension: a cross-sectional study. Int J Cardiovasc Imaging 2020,36: 577584.

19. Muraru D, Badano LP, Nagata Y, et al. Development and prognostic validation of partition values to grade right ventricular dysfunction severity using 3D echocardiography. Eur Heart J Cardiovasc Imaging 2020, 21: 10-21.

20. Huang KC, Lin LY, Chen YS, et al. Three-Dimensional Echocardiography-Derived Right Ventricular Ejection Fraction Correlates with Success of Decannulation and Prognosis in Patients Stabilized by Venoarterial Extracorporeal Life Support. J Am Soc Echocardiogr 2018, 31: 169-179.

21. Kusunose K, Haga A, Abe T, et al. Utilization of Artificial Intelligence in Echocardiography. CIRC J 2019, 83: 1623-1629.

22. Narang A, Mor-Avi V, Prado A, et al. Machine learning based automated dynamic quantification of left heart chamber volumes. Eur Heart J Cardiovasc Imaging 2019, 20: 541-549.

23. Namisaki H, Nabeshima Y, Kitano T, et al. Prognostic Value of the Right Ventricular Ejection Fraction, Assessed by Fully Automated Three-Dimensional Echocardiography: A Direct Comparison of Analyses Using Right Ventricular-Focused Views versus Apical Four-Chamber Views. J Am Soc Echocardiogr. 2021,34:117-126.

24. Medvedofsky D, Mor-Avi V, Kruse E, et al. Quantification of Right Ventricular Size and Function from Contrast-Enhanced Three-Dimensional Echocardiographic Images. J Am Soc Echocardiogr 2017, 30:1193-1202.

25. Pereira JB, Essa M, Ugonabo I, et al. The feasibility of contrast echocardiography in the assessment of right ventricular size and function. Echocardiography. 2019,36:1979-1988.

26. Kuhn A, Meierhofer C, Rutz T, et al. Non-volumetric echocardiographic indices and qualitative assessment of right ventricular systolic function in Ebstein's anomaly: comparison with CMR-derived ejection fraction in 49 patients. Eur Heart J Cardiovasc Imaging 2016,17: 930-935.

27. Ahmad A, Ibrahim Z, Sakr G, et al. A comparison of artificial intelligence-based algorithms for the identification of patients with depressed right ventricular function from 2-dimentional echocardiography parameters and clinical features. Cardiovasc Diagn Ther 2020, 10: 859-868.

28. Liu S, Bose R, Ahmed A, et al. Artificial Intelligence-Based Assessment of Indices of Right Ventricular Function. J Cardiothorac Vasc Anesth 2020, 34: 2698-2702.

Table 1. Clinic Characteristics of the Study Population

\begin{tabular}{ll}
\hline Variable & Patients $(\mathbf{n = 5 1 )}$ \\
\hline Age, median (range) years & $53(34-60)$ \\
Male, n(\%) & $29(56.9)$ \\
Height, cm & $165.5 \pm 8.5$
\end{tabular}




\begin{tabular}{ll}
\hline Variable & Patients $(\mathbf{n = 5 1 )}$ \\
\hline Weight, Kg & $64.9 \pm 11.5$ \\
$\mathrm{BSA}, \mathrm{m}^{2}$ & $1.7 \pm 0.2$ \\
$\mathrm{SBP}, \mathrm{mmHg}$ & $123 \pm 19.3$ \\
$\mathrm{DBP}, \mathrm{mmHg}$ & $77.1 \pm 10.3$ \\
$\mathrm{HR}$, beats/min & \\
During 3DE & $75.7 \pm 11.3$ \\
During CMR & $76.2 \pm 10.1$ \\
Frame rate & $20(16-21)$ \\
Disease Classification & \\
Ischemic heart disease, n (\%) & $13(25.5)$ \\
Hypertrophic cardiomyopathy, n $(\%)$ & $12(23.5)$ \\
Dilated cardiomyopathy, n (\%) & $2(3.9)$ \\
Myocarditis, n (\%) & $2(3.9)$ \\
Normal heart, n (\%) & $14(27.5)$ \\
Others, n (\%) & $8(15.7)$ \\
\hline
\end{tabular}

Categorical variables are expressed as number (\%). Continuous variables that obey the normal distribution are expressed as mean $\pm \mathrm{SD}$. Continuous variables that did not obey the normal distribution are expressed as median (50th) values and interquartile ranges (25th and 75 th).

BSA, Body surface area; DBP, diastolic blood pressure; SBP; systolic blood pressure; HR, heart rate; 3DE, three dimensional echocardiography; CMR, cardiac magnetic resonance.

Table 2. Comparisons of RV volumes and RVEF between AI-based 3DE and CMR

\begin{tabular}{|c|c|c|c|c|c|c|c|c|}
\hline Group & Parameter & AI-3DE & CMR & $\mathrm{P}_{1 \text { Value }}$ & Bias & $\begin{array}{l}\text { LOA } \\
\mathrm{mL}\end{array}$ & $\mathrm{r}$ & $\mathrm{P}_{2}$ Valu \\
\hline \multirow[t]{6}{*}{$\begin{array}{l}\mathrm{ALL} \\
(\mathrm{n}=51)\end{array}$} & $\begin{array}{l}\text { EDV, } \\
\mathrm{ml}\end{array}$ & $\begin{array}{l}98.7 \\
(90.5- \\
128 .)\end{array}$ & $\begin{array}{l}115.4 \\
(92.6- \\
130.2)\end{array}$ & 0.089 & -11.12 & $\begin{array}{l}-62.7- \\
40.5\end{array}$ & 0.61 & $¡ 0.001$ \\
\hline & $\begin{array}{l}\mathrm{EDVi} \\
\mathrm{ml} / \mathrm{m} 2\end{array}$ & $\begin{array}{l}67.5 \\
(55.9- \\
76.4)\end{array}$ & $\begin{array}{l}68.9 \\
(55.9- \\
78.4)\end{array}$ & 0.053 & -6.155 & $\begin{array}{l}-37.6- \\
25.3\end{array}$ & 0.52 & $¡ 0.001$ \\
\hline & $\mathrm{ESV}, \mathrm{ml}$ & $\begin{array}{l}55.8 \\
(46.5- \\
69.3)\end{array}$ & $\begin{array}{l}59.8 \\
(45.2- \\
72.3)\end{array}$ & 0.051 & -3.499 & $\begin{array}{l}-40.0- \\
33.0\end{array}$ & 0.55 & ¡0.001 \\
\hline & $\begin{array}{l}\mathrm{ESVi} \\
\mathrm{ml} / \mathrm{m} 2\end{array}$ & $\begin{array}{l}31.8 \\
(26.6- \\
38.5)\end{array}$ & $\begin{array}{l}34.0 \\
(25.5- \\
42.3)\end{array}$ & 0.572 & -1.285 & $\begin{array}{l}-25.2- \\
22.7\end{array}$ & 0.45 & 0.001 \\
\hline & $\mathrm{SV}, \mathrm{ml}$ & $\begin{array}{l}47.5(37.3- \\
58.6)\end{array}$ & $\begin{array}{l}54.6 \\
(46.2- \\
66.3)\end{array}$ & 0.010 & -7.63 & $\begin{array}{l}-29.2- \\
13.9\end{array}$ & 0.70 & ¡0.001 \\
\hline & $\begin{array}{l}\text { RVEF } \\
\%\end{array}$ & $\begin{array}{l}47 \\
(43-50)\end{array}$ & $\begin{array}{l}51 \\
(44-54)\end{array}$ & 0.077 & -2.167 & $\begin{array}{l}-16.8- \\
12.4\end{array}$ & 0.38 & 0.005 \\
\hline \multirow[t]{2}{*}{$\begin{array}{l}\text { Good } \\
n=18)\end{array}$} & $\begin{array}{l}\text { EDV, } \\
\mathrm{ml}\end{array}$ & $\begin{array}{l}96.6 \\
(84.1- \\
116.5)\end{array}$ & $\begin{array}{l}110.0 \\
(98.0- \\
128.8)\end{array}$ & 0.074 & -15.09 & $\begin{array}{l}-46.5- \\
16.3\end{array}$ & 0.72 & 0.001 \\
\hline & $\begin{array}{l}\text { EDVi, } \\
\mathrm{ml} / \mathrm{m} 2\end{array}$ & $\begin{array}{l}57.4 \\
(50.8- \\
65.4)\end{array}$ & $\begin{array}{l}67.2 \\
(59.8- \\
72.1)\end{array}$ & 0.034 & -8.90 & $\begin{array}{l}-28.4- \\
10.6\end{array}$ & 0.49 & 0.040 \\
\hline
\end{tabular}




\begin{tabular}{|c|c|c|c|c|c|c|c|c|}
\hline Group & Parameter & AI-3DE & CMR & $\mathrm{P}_{1 \text { Value }}$ & Bias & $\begin{array}{l}\mathrm{LOA} \\
\mathrm{mL}\end{array}$ & $\mathrm{r}$ & $\mathrm{P}_{2}$ Valu \\
\hline & $\mathrm{ESV}, \mathrm{ml}$ & $\begin{array}{l}49.6 \\
(44.0- \\
59.2)\end{array}$ & $\begin{array}{l}59.2 \\
(44.4- \\
65.4)\end{array}$ & 0.239 & -7.63 & $\begin{array}{l}-33.7- \\
18.4\end{array}$ & 0.65 & 0.003 \\
\hline & $\begin{array}{l}\mathrm{ESVi} \\
\mathrm{ml} / \mathrm{m} 2\end{array}$ & $\begin{array}{l}30.7 \\
(25.2- \\
33.5)\end{array}$ & $\begin{array}{l}32.5 \\
(27.6- \\
39.8)\end{array}$ & 1.000 & -3.07 & $\begin{array}{l}-25.4- \\
19.2\end{array}$ & 0.47 & 0.052 \\
\hline & $\mathrm{SV}, \mathrm{ml}$ & $\begin{array}{l}46.9 \\
(40.1- \\
56.7)\end{array}$ & $\begin{array}{l}55.3 \\
(47.6- \\
63.8)\end{array}$ & 0.055 & -7.47 & $\begin{array}{l}-23.8- \\
8.84\end{array}$ & 0.74 & $<0.001$ \\
\hline & $\begin{array}{l}\text { RVEF } \\
\%\end{array}$ & $\begin{array}{l}49 \\
(47-54)\end{array}$ & $\begin{array}{l}52 \\
(45-55)\end{array}$ & 0.606 & -0.32 & $\begin{array}{l}-13.6- \\
13.0\end{array}$ & 0.61 & 0.008 \\
\hline \multicolumn{2}{|c|}{$\begin{array}{l}\text { Moderate+PodæDV, ml } \\
(\mathrm{n}=33)\end{array}$} & $\begin{array}{l}104.1(92.3 \\
-136)\end{array}$ & $\begin{array}{l}118.3 \\
(86.1-146.9)\end{array}$ & 0.349 & -8.961 & $-68.8-50.9$ & 0.60 & ¡0.001 \\
\hline & $\begin{array}{l}\mathrm{EDVi} \\
\mathrm{ml} / \mathrm{m} 2\end{array}$ & $\begin{array}{l}62.0 \\
(52.0- \\
74.2)\end{array}$ & $\begin{array}{l}69.6 \\
(47.9- \\
78.2)\end{array}$ & 0.346 & -4.657 & $\begin{array}{l}-40.9- \\
50.9\end{array}$ & 0.52 & 0.002 \\
\hline & $\mathrm{ESV}, \mathrm{ml}$ & $\begin{array}{l}57.7 \\
(47.6 \\
-77.5)\end{array}$ & $\begin{array}{l}63.4 \\
(42.7- \\
73.1)\end{array}$ & 0.964 & -1.247 & $\begin{array}{l}-42.0- \\
39.6\end{array}$ & 0.53 & 0.001 \\
\hline & $\begin{array}{l}\mathrm{ESVi} \\
\mathrm{ml} / \mathrm{m} 2\end{array}$ & $\begin{array}{l}34.7 \\
(28.7- \\
41.4)\end{array}$ & $\begin{array}{l}36.6 \\
(24.7- \\
44.8)\end{array}$ & 0.959 & -0.314 & $\begin{array}{l}-25.2- \\
24.6\end{array}$ & 0.43 & 0.013 \\
\hline & $\mathrm{SV}, \mathrm{ml}$ & $\begin{array}{l}48.3 \\
(37.1 \\
-59)\end{array}$ & $\begin{array}{l}52.6 \\
(45.7- \\
67.8)\end{array}$ & 0.074 & -7.719 & $\begin{array}{l}-31.9- \\
16.5\end{array}$ & 0.69 & ¡0.001 \\
\hline & $\begin{array}{l}\text { RVEF } \\
\%\end{array}$ & $\begin{array}{l}45(42 \\
-49)\end{array}$ & $\begin{array}{l}50 \\
(43-53)\end{array}$ & 0.066 & -3.176 & $\begin{array}{l}-18.3- \\
11.9\end{array}$ & 0.36 & 0.038 \\
\hline
\end{tabular}

Continuous variables that did not obey the normal distribution are expressed as median (50th) values and interquartile ranges $(25$ th -75 th $)$.

RV, right ventricle; RVEF, right ventricular ejection fraction; AI, artificial intelligence; 3DE, threedimensional echocardiography; CMR: Cardiovascular magnetic resonance; EDV, end-diatolic volume; EDVi, RV end-diatolic volume index; ESV, end-systolic volume; ESVi: RV end-systolic volume index; SV, stroke volume; LOA, limits of agreement.

P1 value for AI-3DE versus CMR; P2 value for correlation between AI-3DE and CMR

Table 3. Comparing Volumetric and Non-volumetric Parameters from AI-based 3DE in Identifying CMR-derived RVEF $<45 \%$

\begin{tabular}{|c|c|c|c|}
\hline Variables & CMR-derived RVEF [?]45\% $(n=36)$ & CMR-derived RVEF $\mathbf{i} 45 \%(n=15)$ & $\mathbf{P}$ \\
\hline Age, median (range) years & $54(35-61)$ & $38(29-60)$ & 0.2 \\
\hline Male, $\mathrm{n}(\%)$ & $24(66.7)$ & $5(33.3)$ & \\
\hline Height, cm & $166.9 \pm 8.5$ & $163.3 \pm 7.8$ & \\
\hline Weight, Kg & $65.1 \pm 12.1$ & $64.4 \pm 10.6$ & \\
\hline $\mathrm{BSA}, \mathrm{m}^{2}$ & $1.7 \pm 0.2$ & $1.7 \pm 0.2$ & \\
\hline CMR-derived RVEF, \% & $49(50-55)$ & $40(27-43)$ & \\
\hline
\end{tabular}




\begin{tabular}{llll}
\hline Variables & CMR-derived RVEF [?]45\% (n=36) & CMR-derived RVEF $\mathbf{i 4 5 \%}(\mathbf{n}=\mathbf{1 5})$ & P V \\
\hline EDV, ml & $98.7(88.0-126.5)$ & $101.3(91.4-141.1)$ & 0.77 \\
EDVi, ml/m2 & $59.3(50.3-67.3)$ & $62.0(55.3-80.8)$ & 0.15 \\
ESV, ml & $50.9(45.9-60.5)$ & $65.8(51.7-82.5)^{*}$ & 0.02 \\
ESVi, ml/m2 & $30.7(25.8-36.6)$ & $38.8(31.6-53.4)^{*}$ & 0.00 \\
SV, ml & $50.1(40.0-65.3)$ & $43.9(31.6-50.3)^{*}$ & 0.01 \\
RVEF, $\%$ & $49(45-52)$ & & 0.00 \\
Non-volumetric Parameters & & $39.3(35.0-43.6)$ & 0.16 \\
RVDd base, mm & $36.0(34.3-39.1)$ & $30.9(27.1-39.7)$ & 0.68 \\
RVDd mid, mm & $31.1(28.5-34.8)$ & $76.9(71.5-80.1)$ & 0.07 \\
RVLd, mm & $83.3(76.9-86.1)$ & $15(12.5-18.6)^{*}$ & 0.00 \\
TAPSE, mm & $19.8(17.0-23.2)$ & $37.5(27.3-43.7)^{*}$ & 0.00 \\
FAC, $\%$ & $45.7(40.9-50.1)$ & $12.5(15.7-9.1)^{*}$ & 0.00 \\
RVLS Septum, -\% & $22.8(26.4-14.4)^{*}$ & 0.02 \\
RVLS Freewall, -\% & $19.1(22.5-14.9)$ &
\end{tabular}

Categorical variables are expressed as number (\%). Continuous variables that obey the normal distribution are expressed as mean \pm SD. Continuous variables that did not obey the normal distribution are expressed as median (50th) values and interquartile ranges (25th - 75th).

AI, artificial intelligence; 3DE, three-dimensional echocardiography; CMR: cardiovascular magnetic resonance; EDV, end-diatolic volume; EDVi, RV end-diatolic volume index; ESV, end-systolic volume; ESVi: RV end-systolic volume index; SV, stroke volume; RVEF: right ventricular ejection fraction; TAPSE, tricuspid annular systolic excursion; FAC, fractional area change; RVLS, right ventricle longitudinal strain. $\mathrm{P}$ value for RVEF [?] $45 \%$ versus RVEF ¡45\%.

Table 4. ROC analysis of Multiple Parameters from AI-based 3DE in Diagnosing RV Dysfunction (CMR-derived RVEF $\mathbf{i} 45 \%$ )

\begin{tabular}{|c|c|c|c|c|c|c|c|}
\hline Group & Parameter & AUC & $95 \% \mathrm{CI}$ & P Value & $\begin{array}{l}\text { Cutoff } \\
\text { value }\end{array}$ & Sensitivity & Specificity \\
\hline \multirow[t]{8}{*}{$\begin{array}{l}\text { ALL } \\
(\mathrm{n}=51)\end{array}$} & $\mathrm{ESV}, \mathrm{ml}$ & 0.706 & $\begin{array}{l}0.540- \\
0.872\end{array}$ & 0.022 & 59.9 & $77.8 \%$ & $60 \%$ \\
\hline & $\begin{array}{l}\mathrm{ESVi} \\
\mathrm{ml} / \mathrm{m} 2\end{array}$ & 0.747 & $\begin{array}{l}0.594- \\
0.900\end{array}$ & 0.006 & 38.5 & $75 \%$ & $60 \%$ \\
\hline & $\mathrm{SV}, \mathrm{ml}$ & 0.706 & $\begin{array}{l}0.576- \\
0.865\end{array}$ & 0.021 & 45.9 & $63.9 \%$ & $67.6 \%$ \\
\hline & RVEF, \% & 0.854 & $\begin{array}{l}0.728- \\
0.970\end{array}$ & ¡0.001 & 43 & $94.4 \%$ & $66.6 \%$ \\
\hline & $\begin{array}{l}\text { TAPSE, } \\
\mathrm{mm}\end{array}$ & 0.757 & $\begin{array}{l}0.589- \\
0.926\end{array}$ & 0.004 & 16.3 & $88.9 \%$ & $66.0 \%$ \\
\hline & FAC, $\%$ & 0.779 & $\begin{array}{l}0.627- \\
0.930\end{array}$ & 0.002 & 39.6 & $91.7 \%$ & $60 \%$ \\
\hline & $\begin{array}{l}\text { RVLS } \\
\text { Septum, } \\
-\%\end{array}$ & 0.751 & $\begin{array}{l}0.582- \\
0.920\end{array}$ & 0.005 & -26.6 & $69.4 \%$ & $53.3 \%$ \\
\hline & $\begin{array}{l}\text { RVLS } \\
\text { Freewall, } \\
\text {-\% }\end{array}$ & 0.708 & $\begin{array}{l}0.544- \\
0.873\end{array}$ & 0.020 & -21.6 & $50 \%$ & $53.3 \%$ \\
\hline \multicolumn{2}{|c|}{$\begin{array}{l}\text { Moderate+PooiESV, ml } \\
(\mathrm{n}=33)\end{array}$} & 0.682 & $0.486-0.878$ & 0.093 & - & - & - \\
\hline
\end{tabular}




\begin{tabular}{|c|c|c|c|c|c|c|c|}
\hline Group & Parameter & AUC & $95 \% \mathrm{CI}$ & P Value & $\begin{array}{l}\text { Cutoff } \\
\text { value }\end{array}$ & Sensitivity & Specificity \\
\hline & $\begin{array}{l}\mathrm{ESVi} \\
\mathrm{ml} / \mathrm{m} 2\end{array}$ & 0.742 & $\begin{array}{l}0.560- \\
0.924\end{array}$ & 0.025 & 44.1 & $68.2 \%$ & $63.6 \%$ \\
\hline & $\mathrm{SV}, \mathrm{ml}$ & 0.669 & $\begin{array}{l}0.480- \\
0.859\end{array}$ & 0.117 & - & - & - \\
\hline & RVEF, \% & 0.835 & $\begin{array}{l}0.665- \\
0.941\end{array}$ & 0.002 & 43 & $90.9 \%$ & $72.7 \%$ \\
\hline & $\begin{array}{l}\text { TAPSE, } \\
\mathrm{mm}\end{array}$ & 0.702 & $\begin{array}{l}0.465- \\
0.940\end{array}$ & 0.061 & - & - & - \\
\hline & $\mathrm{FAC}, \%$ & 0.731 & $\begin{array}{l}0.534- \\
0.929\end{array}$ & 0.032 & $38.1 \%$ & $95.5 \%$ & $54.5 \%$ \\
\hline & $\begin{array}{l}\text { RVLS } \\
\text { Septum, } \\
-\%- \\
\% \text { sepSeptum, } \\
-\%\end{array}$ & 0.767 & $\begin{array}{l}0.573- \\
0.960\end{array}$ & 0.014 & -22.6 & $77.3 \%$ & $54.5 \%$ \\
\hline & $\begin{array}{l}\text { RVLS } \\
\text { Freewall, } \\
-\%\end{array}$ & 0.667 & $\begin{array}{l}0.459- \\
0.876\end{array}$ & 0.122 & - & - & - \\
\hline
\end{tabular}

ROC, receiver operating curve; AI, artificial intelligence; 3DE, three-dimensional echocardiography; RV, right ventricle; ESV, end-systolic volume; ESVi: indexed RV end-systolic volume; SV, stroke volume; EF: ejection fraction; TAPSE, tricuspid annular systolic excursion; FAC, fractional area change; RVLS, right ventricle longitudinal strain

Table 5. Inter- and Intra-observer Variability of RV Quantitative Parameters from AI-based $3 \mathrm{DE}(\mathrm{n}=20)$

\begin{tabular}{|c|c|c|c|c|}
\hline Characteristics & Inter-observer variability & Inter-observer variability & Inter-observer variability & Intra-c \\
\hline & ICC & 95\% Confidence Interval & $\mathrm{P}$ value & ICC \\
\hline \multicolumn{5}{|c|}{ Volumetric Parameters } \\
\hline $\mathrm{EDV}, \mathrm{ml}$ & 0.866 & $0.693-0.945$ & ¡0.001 & 0.984 \\
\hline $\mathrm{EDVi}, \mathrm{ml} / \mathrm{m}^{2}$ & 0.768 & $0.502-0.901$ & ¡0.001 & 0.872 \\
\hline $\mathrm{ESV}, \mathrm{ml}$ & 0.840 & $0.736-0.906$ & ¡0.001 & 0.803 \\
\hline $\mathrm{ESVi}, \mathrm{ml} / \mathrm{m}^{2}$ & 0.801 & $0.676-0.881$ & ¡0.001 & 0.829 \\
\hline $\mathrm{SV}, \mathrm{ml}$ & 0.864 & $0.688-0.944$ & ¡0.001 & 0.796 \\
\hline RVEF, \% & 0.812 & $0.693-0.888$ & ¡0.001 & 0.814 \\
\hline \multicolumn{5}{|c|}{ Non-volumetric Parameters } \\
\hline RVDd base, mm & 0.848 & $0.657-0.937$ & ¡0.001 & 0.870 \\
\hline RVDd mid, mm & 0.831 & $0.621-0.929$ & $¡ 0.001$ & 0.859 \\
\hline RVLd, mm & 0.827 & $0.613-0.928$ & $¡ 0.001$ & 0.838 \\
\hline TAPS, mm & 0.824 & $0.608-0.926$ & ¡0.001 & 0.870 \\
\hline FAC, $\%$ & 0.875 & $0.792-0.927$ & ¡0.001 & 0.808 \\
\hline RVLS Septum, -\% & 0.926 & $0.822-0.970$ & ¡0.001 & 0.947 \\
\hline RVLS Freewall, -\% & 0.824 & $0.711-0.896$ & ¡0.001 & 0.886 \\
\hline
\end{tabular}

$\mathrm{AI}$, artificial intelligence; 3DE, three-dimensional echocardiography; EDV, end-diatolic volume; EDVi, indexed sum of RV end-diatolic volume; ESV, end-systolic volume; ESVi: indexed RV end-systolic volume; 
SV, stroke volume; EF: ejection fraction; TAPSE, tricuspid annular systolic excursion; FAC, fractional area change; RVLS, right ventricle longitudinal strain

Figure Legends

Figure 1. Representative fully automated analysis of RV size and function by AI-based 3DE. (a) RV volumetric parameters, including EDV, EDVi, ESV, ESVi, SV and RVEF were automatically calculated after tracing the RV endocardium throughout the cardiac cycle in multiple tomographic views; (b) the conventional 2D RV non-volumetric parameters, including TAPSE, FAC, RV septum LS, and RV free-wall LS were presented automatically side by side.

AI-based 3DE: artificial intelligence-based three-dimensional echocardiography; RV: right ventricle; EDV: end-diastolic volume; EDVi: end-diastolic volume index; ESV: end-systolic volume; ESVi: end-systolic volume index; SV: stroke volume; RVEF: right ventricular ejection fraction; 2D: two dimension; TAPSE: tricuspid annular systolic excursion; FAC:fraction area change; LS: longitudinal strain.

Figure 2. Comparison between AI-based 3DE and CMR measurements of RV volumes and EF: linear regression (a) and Bland-Altman analysis (b) for EDV (a1 and b1), EDVi (a2 and b2), ESV (a3 and b3), ESVi (a4 and b4), SV (a5 and b5) and EF (a6 and b6). On the Figure a, the black solid lines represent the regression lines of all measurements, and the black dotted lines indicate $\mathrm{Y}=\mathrm{X}$. On the Figure $\mathrm{b}$, the red dashed lines indicate the Bias, and the black dotted lines indicate the 95\% LOA.

AI-based 3DE: artificial intelligence-based three-dimensional echocardiography; CMR, cardiac magnetic resonance; RV: right ventricle; EDV: end-diatolic volume; EDVi: end-diastolic volume index; ESV: end-systolic volume; ESVi: end-systolic volume index; SV: stroke volume; EF: ejection fraction; LOA:On the left panels

Figure 3. Receiver operator curves (ROCs) representing univariate analysis of multiple parameters from AI-based 3DE in diagnosing RV Dysfunction (CMR-RVEF;45\%). Odds ratios and area under the curve (AUC) values were calculated for each of the variables of interest, along with 95\% CIs.

AI-based 3DE: artificial intelligence-based three-dimensional echocardiography; CMR, cardiac magnetic resonance; RV: right ventricle; ESVi: end-systolic volume index; FAC, fractional area change; EF: ejection fraction; LS: longitudinal strain.

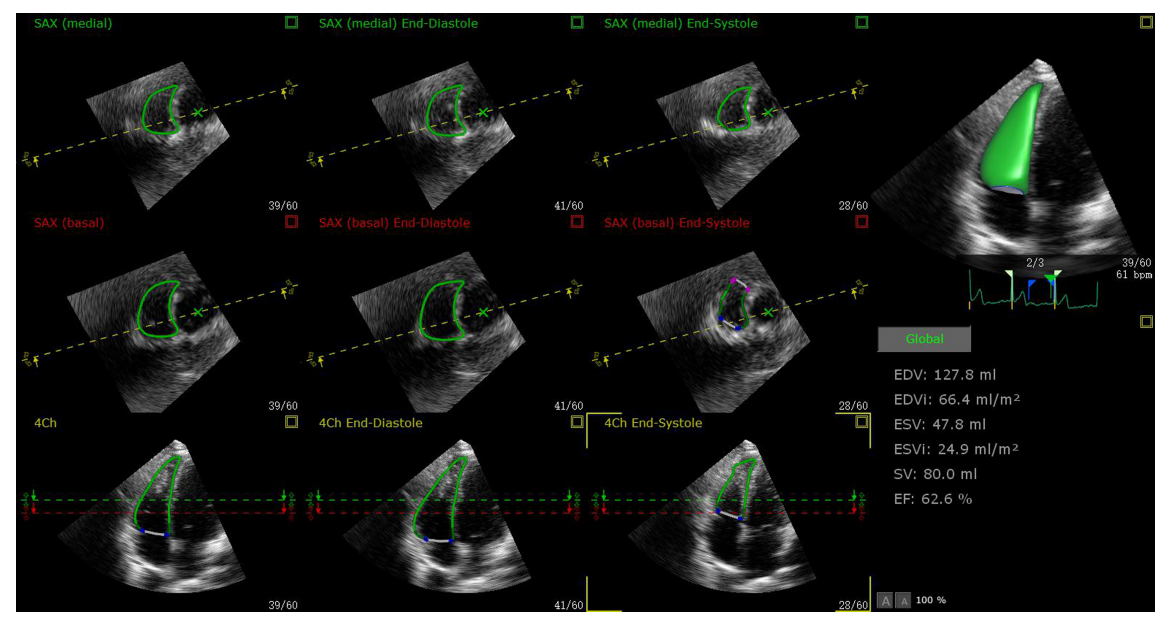



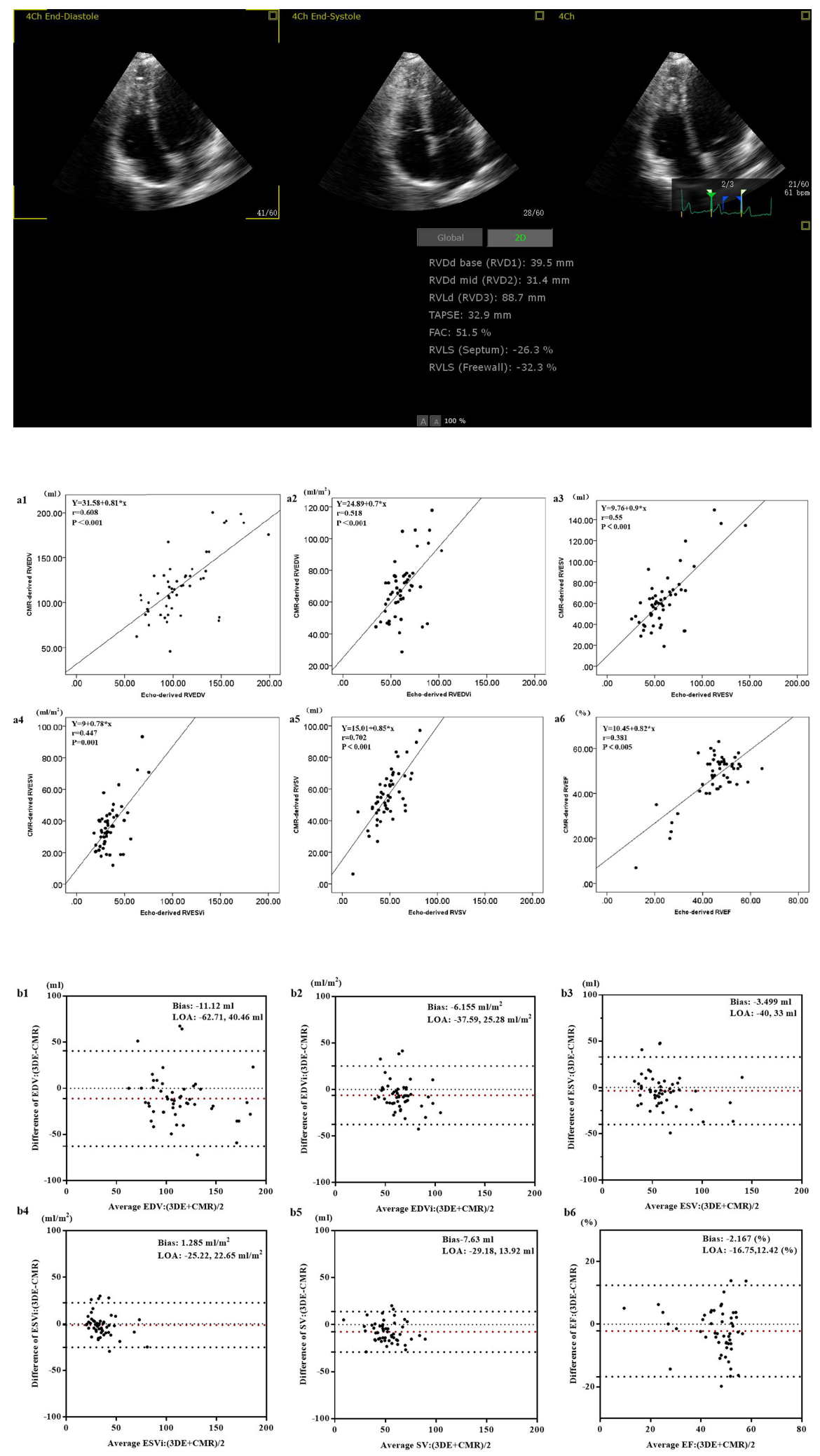


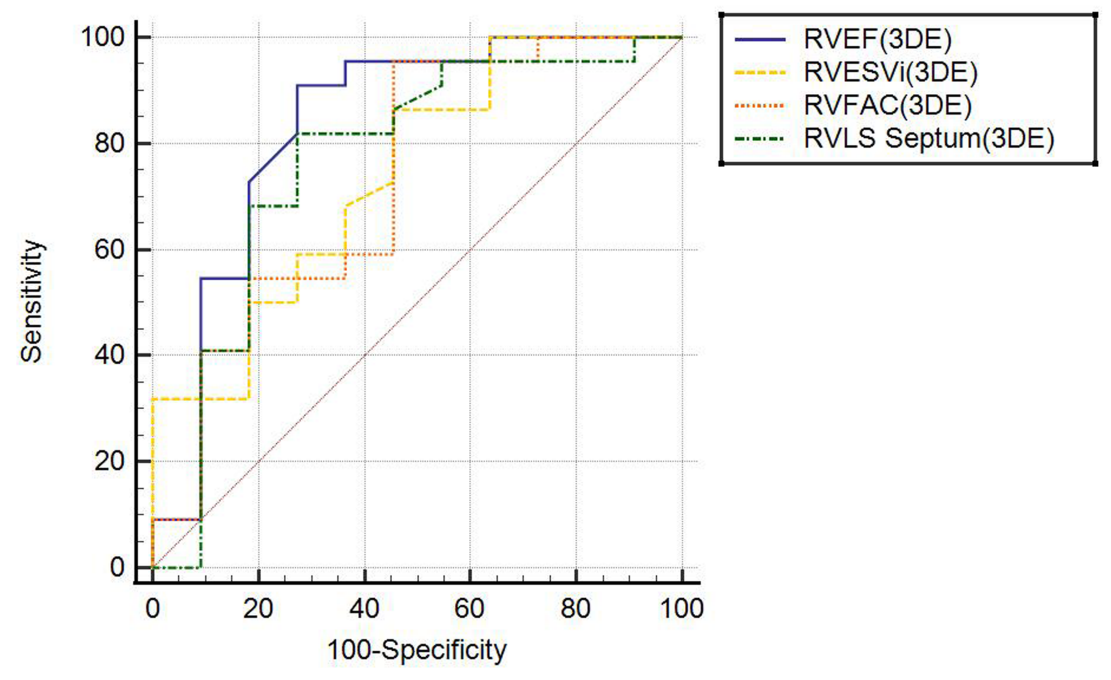

